\title{
New Systematic Solution for Resolving Nonlinear Dynamics Using System Analytical Theory Based on Engineering Science
}

\author{
Cha Deok-soo ${ }^{1} \&$ Kim Kyoung-il ${ }^{2}$ \\ ${ }^{1}$ Eho tech Co. Research center, Soong-sil University, South Korea \\ ${ }^{2}$ Chung-ang University, Electrical Power Division, Seoul, South Korea \\ Correspondence: Cha Deok-soo, Eho tech Co. Research center, Soong-sil University, South Korea.
}

Received: October 9, 2021

Accepted: November 2, 2021

Online Published: November 9, 2021

doi:10.5539/mas.v15n6p46

URL: https://doi.org/10.5539/mas.v15n6p46

\begin{abstract}
There are many nonlinear dynamics in field of non-physical sciences, such as the food chain, economic systems, or engineering systems with the characteristics of closed or open-loop systems. The problems arising from this have been resolved by the outdated chaos theory in statistical physics based on the paradigm of logical thinking. However, it was founded by classical physicists, and it is imperfect and vague, moreover, very difficult for others. Therefore, we require a perfect systematic solution based on systems thinking, such as systems analytical methods in engineering science. Surprisingly, in 2021, a non-physicist, on behalf of a physicist, has successfully resolved the problems and achieved a new solution based on systems thinking through interdisciplinary research; moreover, it has been published. Unfortunately, most physicists do not welcome it because they have no experience and it is disadvantageous to them like the Copernican theory. In addition, they have no ability to evaluate the new solution because they do not know the analytic method. Nevertheless, non-physicists are greatly welcome it, thus, there is no problem in it. Hence, non-physicists will verify it using MATLAB or simulator and apply it to all science, on behalf of physicists. If so, non-physicists will have both a logical solution and a systematic solution for resolving nonlinear dynamics.
\end{abstract}

Keywords: nonlinear dynamics, control theory, MATLAB, systems thinking

\section{Introduction}

This article will detail to the readers how to resolve nonlinear dynamic systematic problems using systems analytical methods in engineering science. Therefore, this is [an interdisciplinary science] between physics and engineering". Remarkably, it has been introduced the engineering principle into traditional physics, further, this solution has been succeed in resolving it and achieved a new solution by a non-physicist on behalf of a physicist in 2021, which is published it previously. (Cha, D. S. \& Kim, K. I., 2021) Hence, this article may not be reviewed and evaluated by physicists, because physicists have not known the engineering principle. Therefore, this article is written for non-physicists in other sciences. Meanwhile, such topics are classified into physical science, but this article is interdisciplinary researches; it is resolved the physical problems such as nonlinear dynamics using systems analytical methods in engineering science. Hence, this article can be reviewed and evaluated by non-physicists, mathematicians or engineers on behalf of physicists if physicists avoid verifying the new solution; there is no problem. Thus, it would be a revolutionary scientific research result, such as Einstein's relativity theory. Accordingly, it will cover nonlinear dynamics including complex systems and metaphysical problems in modern science. Meanwhile, the author summarized the above-mentioned report as follow.

a) Traditional physics has an old solution for nonlinear dynamics, such as the chaos theory. However, physicists do not welcome it because it is imperfect, vague, and difficult for others.

b) Classical physics approached many unsolved nonlinear dynamic systematic problems such as ecosystems with logical thinking with macroscopic linear static viewpoints and defined as a logical problem.

c) Subsequently, they defined it as a black box, as shown in Figure 1(b). However, this is not a reasonable idea because most nonlinear systematic problems are characterized as closed loop systems, as shown in Figure 1(c) above. 
d) A non-physicist has successfully resolved these problems through experimentation on behalf of physicists using systems analysis theory (Golnaraghi, F. \& Kuo, B. C., 1998), as shown in Figure 1(a).

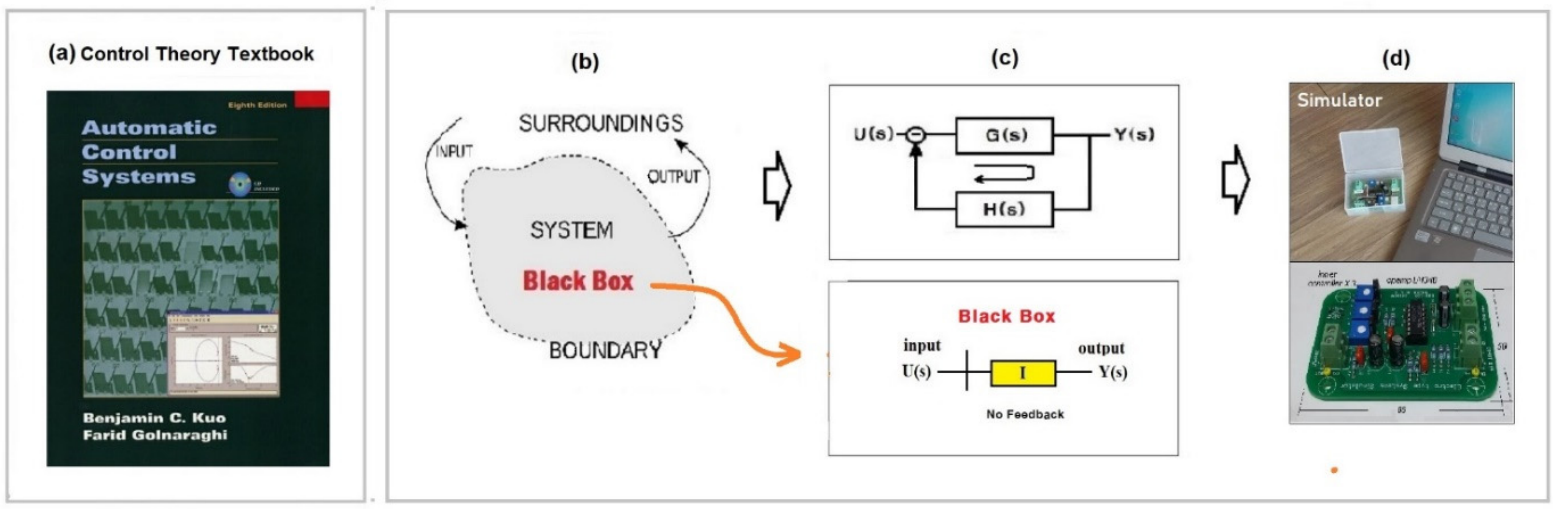

Figure 1. (a) control theory textbook (b) Basic system (c) Inrenal structure in closed loop systems (d) A analog simulator for nonlinear dynamics

In other words, modern physics has approached physical problems algebraically or logically; otherwise, the author has approached it systematically. Moreover, it has been proved experimentally through simulation, as shown in Figure 1(d); no one can fool else. Therefore, there is no choice for determinists. Unfortunately, classical physicists in the $17^{\text {th }}$ century have no solution, such as the above-mentioned systems analytical method in control theory. Hence, they developed chaos theory using algebra and statistics. Physicists do not know the systems analysis method in control theory but, non-physicists welcome it because the new solution is more perfect and precise compared to the primitive chaos theory.

Unfortunately, there is a serious problem. Most physicists strongly deny and reject this systematic solution; moreover, even the author is hostile. Why do they so? Because there is already a chaos theory that they are using. However, there is other reason; it is very disadvantageous to them, like Galileo's heliocentric theory. If this is true, they would lose everything like medieval astronomers. Next, they have no ability to review and evaluate the new systematic solution because they have no knowledge with control theory in engineering science. So, they have no way of refuting or criticizing the research results. Thus, physicists are unconditionally opposed to them without reason. Moreover, all of them wish to stay in a safe zone as outsiders because they have the old solution such as chaos theory. No scientists want to innovate in their discipline. Nevertheless, it is their own problem, hence, it has nothing to do with non-physicists, such as a physicist who rejects the heliocentric theory, an automobile engineer who avoids electric vehicles, and a surgeon who opposes the emergence of a physician. Therefore, the author has no reason to entreat adoption to them.

Meanwhile, non-physicists have no obligation to follow determinists and their chaos theory in their statistical physics in determinism. If they intend to hide their shortcomings from other scientists, they would lose their credibility because it is to deceive other scientists and the public. It will be a risk that they would lose their trust in the future if other scientists understand their problems. Nevertheless, they devalued the new solution as an epistemological problem; moreover, they expected the author to fail. Therefore, some physical journals are unfriendly to authors and reject the submission. In addition, they intentionally avoid collaborating with engineering science through interdisciplinary research. As the result, the author understood that it was impossible to contribute to a physics journal and the author failed to convince physicists in modern science, there is a need to find another way to apply to other sciences without resistances.

Finally, the authors will work to ensure that the new solutions are verified and established by non-physicists, and non-physicists are encouraged to participate in the study. To this end, the author presents practical application examples for benchmarking in Section 2 of this report. Accordingly, this report contributes to the advancement of modern science.

\section{Materials and Method}

\subsection{Scientific Background}

Meanwhile, both classical and modern physicists have divided them into open loop systems, such as logical problems and closed loop systems, including systematic problems. Even though there seem to be no problems externally, there are serious problems hidden. Determinists have treated these systematic problems as logical problems. Accordingly, they treated all physical phenomena as a black box, as shown in Figure 1(b). Then, they 
resolved it using logical solutions, such as chaos theory in statistical physics. Unfortunately, chaos theory is not a perfect systematic solution, which was found using algebra and statistics by classical physicists a long time ago. It is imperfect and difficult to study for others, but other scientists have no choice. Hence, we need a perfect solution for resolving nonlinear dynamic systems because determinists have no systematic solution within closed loop systems with feedback.

In 2021, amazingly, a non-physicist on behalf of a physicist discovered a mathematical tool for resolving nonlinear dynamic systems such as system analytical methods in adjacent disciplines, as shown in Figure 1(a). It seems shocking to readers because it has already been used in engineering science. Classical physicists ignore the control theory. It was developed in the $20^{\text {th }}$ century for designing automatic systems in industrial plants by engineering scientists such as Nyquist (1976), but determinists avoid cooperating with other science because they have the primitive chaos theory. If they collaborate with engineering science through interdisciplinary research, they could resolve their nonlinear dynamic systematic problems, such as ecosystems. For a more detailed description, refer to references (Cha, D. S., 2015), (Cha, D. S. \& Jun, H. J., 2020).

\subsection{Overview of New Solution}

For instance, in food chain in ecosystems, assuming it has are predators with positive entropy and prey with negative entropy, the amount entropy must be converged to zero state (equilibrium) by time based on the energy conservation. We can convert the food chains into a closed loop system as shown in Figure 1(c) above, because ecosystems are a circulation system eternally. This is the main concept in this research report. Accordingly, if the rainfall in the grassland changes seasonally, a number of deer and wolves will fluctuate continuously as shown in Figure 2, it can be simulated with computer or analog simulator as shown in Figure 1(d); please the readers watch the video clip (YouTube, 2008). In this case, we can observe the behavior of complexity. (Casti, J. L., 1995) In nonlinear dynamics from Figure 2, which is included irregularity, regularity, self-organizations and initial-phenomenon.

Surprisingly, if anyone who are not know chaos theory is able to transform the stock market or thermodynamics into closed loop system, they can easily resolve nonlinear dynamics using simulation. Thus, we need not primitive chaos theory no more. Unfortunately, non-physicists except control engineers would be very difficult the control theory, but there is no problem. All scientists should be supported by computer MATLAB (Mathworks co., 2021).

or analog simulator as shown in Figure 1(d). Paradoxically, if engineers had developed control theory including systems analytical methods in the $17^{\text {th }}$ century; moreover, classical physicists had learned the methods, they might resolve completely the nonlinear dynamics including metaphysical problems based on the paradigm of systems thinking (Corporate finance institute, 2015). However, physicists avoid cooperating with engineering science, it is self-deception. Because they need not to be supported by other discipline and collaborated with other scientists. Non-physicists cannot agree with their idea.

Again, if any scientists can be transformed any unsolved nonlinear dynamic problems such as ecosystems into closed loop system with feedback as shown in Figure 1(c), any scientists can resolve such problems in in their discipline. They must keep in mind that nonlinear dynamics is not logical problems but systematic problems. In addition, there is something to note; most systematic problems in scientific field are impossible to solve algebraically or logically because it has inherent characteristics of feedback, hence, no one can calculate inversely (non-reversibility). Hence it should be followed by following four steps; modeling - simulation verification - return. If anyone cannot understand, please refer to report (Cha, D. S. \& Kim, K. I., 2021).

\subsection{Proof of Imperfection of the Old Chaos Theory}

Meanwhile, the author has failed persuade physicists, and some physical journals were hostile without reason. Unfortunately, they do not want to innovate their discipline such as Copernican theory, they are very conservative discipline; moreover, they have no the ability to review and evaluate the new solution, further, they are extremely reluctant to expose the shortcomings of physics. Nevertheless, non-physicists have no obligation to follow a primitive chaos theory based on logical thinking if they hide their mistakes. Regrettably, this only identified where the shortcomings of physics were. This article will prove the reason why chaos theory is imperfect. The primitive chaos theory is founded algebra and statistics based on logical thinking. 
Table 1. Benchmarking between classical physical law and Application examples

\begin{tabular}{lll}
\hline Example & Before & After (Redefined) \\
\hline Lorenz's butterfly effect & Rapid increasing phenomena & A time series function (Initial phenomena) \\
Random walks in stock market & Irregularity and non-predicable & A time series function (Complexity) \\
logistic curve in ecosystems & Sigmoid Curve & A time series function $y(t)=1-w t)$ \\
Kuhn's innovation theory & Resolution - Paradigm shift - Normal science & A time series function $\left.y(t)=1-\mathrm{e}^{-\mathrm{x} \cdot \mathrm{t}}\right)$ \\
\hline
\end{tabular}

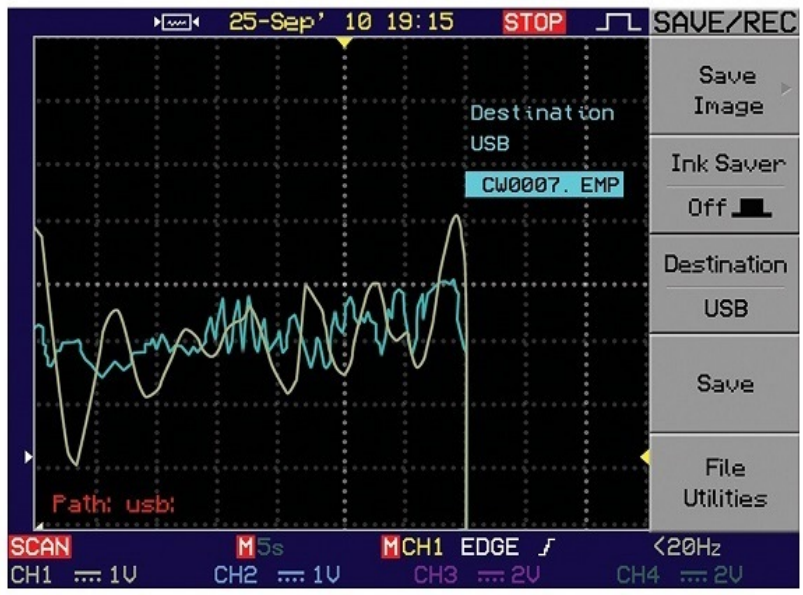

Figure 2. Computer screen; behavior of output of nonlinear dynamics systems (blue line is input, yellow line is output) Video clip; ULR https://www.youtube.com/watch?v=-EnU4L5uH5o

Perhaps, determinists have no idea about the paradigm of systems thinking, so that they cannot approach the metaphysical problem such as 'Why cannot predict the daily stock price? Historically, why do epidemics recur periodically?' In the other academic viewpoints, they have only [vertical thinking] without [horizontal thinking] (Monday Morning Memo, 2008).

(Benchmarking); in this Section, to prove this, we will benchmark it with existing scientific theories. Thus, the author cites a previously published paper of application examples as shown in Table 1. (Cha, D. S. \& Kim, K. I., 2021) Most non-physicists feel to unclear the various laws of physics perfected by classical physicists, no one object to determinists until today. For example, the butterfly effect. (Lorenz, E., 1963), logistic curve. (Linacre, L. J., 2009), random walk (Encyclopedia of Mathematics, 2020), and Kuhn's innovation theory in Table 1, they are laws of physics that physicists are proud of. We will benchmark such classical theories.

However, these examples are very vague and difficult to others but no one doubted it for a long time. As the results, these examples are not physical laws but simple time series functions in nature as shown in Table 1. In other words, we must define these problems as closed loop systemic problems and approaching them with systems thinking. So, there is no academic value but the physicists are misunderstood. Again, all of them are only time series functions with the characteristics of a very simple closed-loop system. In other words, it must be resolved again systematically. Thus, if anyone has a question on Table 1, it is welcomed.

In another case, all scientists have misunderstood that chaos theory in statistical physics was perfect. We need to benchmark their books. The one is [Simply Complexity] of physicist Neil Johnson (Neil, J., 1920) and the other is [Entropy] of economist Jeffrey Johnson (Jeremy, R., 1981). However, they approached nonlinear dynamics based on logical thinking with macroscopic linear static viewpoints. As the results, they found out the output as shown in Figure 2. Amazingly, it realizes complexity including irregularity, regularity, self-organization, and initial phenomena through them. However, they did not find out that it is never runaway, overflowing, or exploding because they do not know the inherent characteristics of closed loop systems. Paradoxically, if they have known the inherent characteristics in the systems, they would not written such a book.

In practice case, to help the readers understand, this section presents [Adam Smith's Invisible hand] as an example. We can transform a market into Figure 1(c); it has supplier $\mathrm{Q}(\mathrm{s})$ and a demander $\mathrm{H}(\mathrm{s})$. If $\mathrm{Q}(\mathrm{s})$ is increasing the supplying volume but the system presses price lower. Likewise, if $\mathrm{H}(\mathrm{s})$ is increasing demanding volume but the system presses price upper. In other words, demander $\mathrm{H}(\mathrm{s})$ is a negative feedback element, which is prevent the price to increase rapidly. There is an invisible power in it, hence, it is converged equilibrium by 
self-controlled. Remarkably, we called it as Adam Smith's invisible hand. In addition, it is same as the law of supply and demand. (Raphael, Z., 2014). As the result, we can transform and build a model system such as Figure 1(c), we can reproduce the behavior of output using simulator. The output is determined Equation (1) as below. In addition, its practical the output is shown in Figure 2; it is realizing nonlinear dynamics.

$$
y(t)=1-A \cdot e^{-B \cdot t} \sin (W \cdot t+\varphi)
$$

Where, $\mathrm{y}(\mathrm{t})$; output, $\mathrm{t}$; time, A, B, W, $\varphi$; Variable constant. If so, economists need not hard work because they can analyze to simulate their economic problems such as macroeconomics.

\section{Results}

Summarized the above description, (1) if we compared the old solution with the new solution, we can understand that the old chaos theory is resolved algebraically (logically) but the new solution is solved systematically. (2) Unfortunately, physicists have no ability to resolve the systematic problems based on systems thinking because they have no experience about control theory. (3) Therefore, non-physicists have no choice but to work on behalf of physicists, and, they have been resolved successfully and achieved new solution. (4) Accordingly, non-physicists have no reason to object it. Nevertheless, physicists already do not welcome it because they have had the primitive chaos theory; moreover, they do not want to study about interdisciplinary research. (5) Regrettably, the author has failed to convince them to the end, hence, we need to find another way to verify it and establish to modern science.

\section{Discussions}

Meanwhile, this article provides a systematic solution for resolving nonlinear dynamics for the first time, but most physicists do not welcome it. Why do they do so? In practice, the reason is that they have no ability to review and evaluate this new solution. If so, they might think of it as insulting themselves. Therefore, they asserted that the new solution is an epistemological problem that no one can understand. In medieval age, if the Galileo on behalf of professional astronomers found the Earth is round, it means that professional astronomers have no capability. Likewise, determinists avoid more advanced new systematic solution because they do not want to be involved in the risk because they have already the old solution such as chaos theory.

Therefore, they do not want to appear another advanced solution, as mentioned above; that it is disadvantageous to them. It is similar to medieval surgeons who oppose the emergence of internists with X-rays and stethoscopes. Therefore, it is their own problem, and it has nothing to do with non-physicists, who has no reason to plead with them.

Meanwhile, there is a considerable problem regarding the paradigm of scientific thinking. If physicists' ultimate goal is perfect physical science, they should approach physical phenomena based on both logical thinking and systems thinking; [logical thinking + systems thinking]. In this case, we need to introduce the systems analytical method in control theory into physical science via interdisciplinary science in the future. It is an excellent idea without risk to physicists. In this case, other scientists have no reason to object to it. However, all scientists need to learn the systems analysis theory in control theory. Since it is as complex as chaos theory, scientists must be supported by the program MATLAB and analog simulator, as shown in Figure 1(d). Paradoxically, if non-physicists mastered the new solution, other scientists could cover non-linear dynamics problems and metaphysical problems in all sciences using the new method on behalf of physicists.

\section{Conclusions}

This article is not written for physicists but other scientists, so that physicists cannot criticize this study. This article is an interdisciplinary research between physics and engineering; moreover, it is introduced the new systematic analytical method into physics for resolving nonlinear dynamics. Accordingly, it will be resolved many unsolved nonlinear dynamic systematic problems involving complexity such as ecosystems in science systematically. Amazingly, the advanced systematic solution has been achieved based on systems thinking by non-physicists on behalf of physicists. Furthermore, it has proved and verified by experiment using computer or simulator, no one can doubt it. Naturally, there is no scientist would know a common sense; physical sciences consist of logical and systematic problems. However, determinists cannot be solved by logical solutions alone. It is like one eyed physics with handicap. Unfortunately, physicists do not welcome the new systematic solution for nonlinear dynamics, but there is no problem. If all scientists have obtained both solutions; moreover, they can compare with both the old and new solution in the future. It would be more advanced solution rather than the old chaos theory. If so, physicists cannot remain outsiders any more. Finally, the author expects to establish in modern science, and will develop into a perfect science with logical and systematic solutions. 
Contributions: Cha, conceptualization and writing, Kim, verifying the systems analysis process.

Deceleration: there is no Competing of Interests.

\section{References}

Cha, D. S., \& Kim, K. I. (2021). New Systems Solution for Resolving Nonlinear Dynamics Based on Systems Thinking. Open Journal of Applied Sciences, 11(10), 1177-1189. https://doi.org/10.4236/ojapps.2021.1110088

Golnaraghi, F., \& Kuo, B. C. (1998). Automatic Control Systems (10th ed.). McGraw-Hill Education 2017.

Cha, D. S. (2015). Establishment of New Solution for Complex Systems in Multidisciplinary Science Based on Feedback System Analysis Method and Proven by Simulator. Open Journal of Modern Physics, 6(13), 1927-1934. https://doi.org/10.4236/jmp.2015.613198

Cha, D., \& Jun, H. (2020). The Origin of Nonlinear Dynamics Involving Complexity in Modern Sciences. Open Journal of Applied Sciences, 10, 654-662. Retrieved from https://www.scirp.org/journal/paperinformation.aspx?paperid=103755

You Tube Video clip. (2008). Retrieved from https://www.youtube.com/watch?v=-EnU4L5uH5o

Casti, J. L. (1995). Complexification: explaining a paradoxical world through the science of surprise. Harper Perennial.

MATLAB. $\quad$ (2021). $\quad$ Retrieved from https://kr.mathworks.com/support/learn-with-matlab-tutorials.html?s_tid=srchtitle

$\begin{array}{llll}\text { Systems } & \text { thinking. } & \text { (2015). } & \text { Retrieved }\end{array}$ https://corporatefinanceinstitute.com/resources/knowledge/strategy/systems-thinking/

$\begin{array}{llll}\text { Horizontal thinking. } & \text { (2008). } & \text { Retrieved }\end{array}$ https://www.mondaymorningmemo.com/newsletters/horizontal-thinking/

Lorenz, E. (1963). Deterministic Nonperiodic Flow. Journal of the atmospheric sciences, 20(2), 130-141. https://doi.org/10.1175/1520-0469(1963)020<0130:DNF $>2.0 . C O ; 2$

Linacre, L. J. (2009). Why Logistic (Sigmoid) Ogive and not Autocatalytic Curve? Retrieved from https://rasch.org/rmt/rmt64k.htm

Random walk. (2020). Encyclopedia of Mathematics. Retrieved from https://encyclopediaofmath.org/wiki/Random_walk

Jeremy, R. (1981). Entropy: A New World View. Bantam Books, London.

Neil, J. (2020). Simply Complexity (2007 Korea ed.). Bada Publishing, Seoul.

Raphael, Z. (2014). The Law of Supply and Demand. Quickonomics.

\section{Copyrights}

Copyright for this article is retained by the author(s), with first publication rights granted to the journal.

This is an open-access article distributed under the terms and conditions of the Creative Commons Attribution license (http://creativecommons.org/licenses/by/4.0/). 